\title{
Insomnia in Perimenopausal Women: A Study at TuDu Hospital, Vietnam
}

\author{
Tuan Vo ${ }^{1}$, Thang Huynh ${ }^{2}$ \\ ${ }^{1}$ Department of Obstetrics and Gynecology, University of Medicine and Pharmacy, Ho Chi Minh City, Vietnam \\ ${ }^{2}$ Department of General Gynecology, Tudu Hospital, Ho Chi Minh City, Vietnam
}

Email address:

vominhtuan@ump.edu.vn (T. Vo)

\section{To cite this article:}

Tuan Vo, Thang Huynh. Insomnia in Perimenopausal Women: A Study at TuDu Hospital, Vietnam. Journal of Gynecology and Obstetrics. Vol. 7, No. 4, 2019, pp. 104-108. doi: 10.11648/j.jgo.20190704.12

Received: May 20, 2019; Accepted: June 19, 2019; Published: June 29, 2019

\begin{abstract}
Insomnia with direct or indirect influences on perimenopausal illnesses was recorded in various studies. In order to improve the quality of treatment, a study is needed to define insomnia prevalence and related factors in perimenopausal clients at Tu Du Ob/Gyn Hospital. A cross-sectional study was conducted during October - December 2017 in 45-to-52-yearsold clients seeking routine gynecology examination at Tu Du Hospital. Insomnia-disorder scoring from Diagnostic and Statistical Manual of Mental Disorders (DSM-5) was used to screen insomnia. Interviews were made on household, sociology, history of perimenopausal illness, economic conditions, marital status, family and social relationship, which, then, was compared between the two groups with diagnostic screening as having insomnia versus no insomnia. 386 cases were admitted into the study, the prevalence of insomina was $27.72 \%$. Insomnia related to age when comparing age groups of 50-52 against 45-49 $(\mathrm{PR}=1.9 ; 95 \% \mathrm{CI}=1.1-3.5)$, economic status $(\mathrm{PR}=2.99 ; 95 \% \mathrm{CI}=1.2-7.6)$, hot flush $(\mathrm{PR}=3.65 ; 95 \% \mathrm{CI}=2-6.6)$. Those findings indicated that Perimenopausal women should be screened on insomnia for counseling and early treatment.
\end{abstract}

Keywords: Perimenopausal, Insomnia, Vietnam

\section{Introduction}

$\mathrm{TuDu}$ is an $\mathrm{Ob} / \mathrm{Gyn}$ teaching hospital (with 2, 500 patient beds) of the central level providing treatment, training and research services. The hospital is appointed by the Ministry of Health as the highest level of referral for 32 provinces/towns in the southern Vietnam. Perimenopausal women is a significant number of clients sought clinical examination, and most of lower level hospitals will refer it to $\mathrm{TuDu}$ hospital for management. Therefore, the number of perimenopausal clients at $\mathrm{TuDu}$ Hospital is regarded as representative of this type of perimenopuasal illness in the southern Vietnam.

Insomnia increases the risk of mortality, the utilization of medical services and the risk of mental disorders, especially depression. Insomnia directly or indirectly affects perimenopausal illnesses [1-3]. The rate of insomnia in perimenopausal women is about 30\% [4-6]. Study results showed a correlation between risk factors and insomnia in perimenopausal women. Therefore, perimenopausal clients should be screened on insomnia when they contact medical staff to plan a therapy for the improved quality of life.

Gold standard for insomnia diagnosis is polysomnography. However, it is difficult to apply this technique in nonpsychiatric facilities. At present, DSM-5 insomnia criteria are possibly applied by non-psychiatric physicians to diagnose insomnia for patients [7-8].

The rate of insomnia is so varying and dependent upon study population and assessment tools. Insomnia rates in perimenopausal women by some studies ranged from $19.7 \%$ to $62.5 \%$ [4-5, 9-13]. As a result, the application of Insomnia disorder scoring-items from DSM-5 criteria for insomnia diagnosis would help to better distinguish between insomnia rate and the rate of sleep quality reduction [8].

Since there are multiple factors that influence the quality of sleep, it is necessary to analyze real impacts of those factors in the population. In Viet Nam, there have not been 
many studies on insomnia yet, especially insomnia in perimenopausal women. Therefore, we conducted the study of "Insomnia rate and related factors in perimenopausal clients who visited Tu Du Hospital for medical checkup" to answer a research question: "What is insomnia prevalence in perimenopausal clients who are seeking medical services at Tu Du Hospital?"

Our study's aims: (1) To determine insomnia prevalence through screening diagnosis clients who sought routine gynecology examination at Tu Du Hospital in the period of October - December 2017.

(2) To examine insomnia-related factors in clients who obtain routine gynecology examination at $\mathrm{Tu}$ Du Hospital.

\section{Subjects and Methods}

\subsection{Patients}

This study was designed as a Cross-sectional study. From October to December 2017, women in the age of 45-52 years old sought routine examination at the Gynecologic Clinic were recruited at Tu Du Hospital. Including subjects should have intact uterus and one or two ovaries, no hormonal therapy within the last three months and agreed to join the study. Our exclusion criterias included: (1) pregnant women, (2) poor physical and mental health status for answering questionnaires.

Sample size calculation was based on the formula of estimating a proportion in a population with absolute precision. With $\mathrm{Z}_{1-\alpha / 2}=1,96 ; \mathrm{d}=0.05 ;(\mathrm{p})$ insomnia prevalence had varied between $19.7 \%$ and $62.5 \%$ as previous studies [9-10, 12-13], hence, we selected $\mathrm{p}=0.50$ to obtain the largest sample size, $\mathrm{n}=386$.

From Oct 2017 to Dec 2017, 4000 perimenopausal women were visited to Gynecology clinic of TuDu Hospital. Base on inclusion criterias, we recruited study subjects randomly. 368 satisfied clients enrolled into the research voluntarily $(9.65 \%$ of 4000).

\subsection{Study Procedures}

Step 1: Questionnaire testing (Pilot study)

Conducting interview with ten women who came for routine gynecology examination to adjust the questionnaire for better fit and communication. Pilot information was not input into the study.

Step 2: Screening and admitting study subjects

At the reception room of the Tu Du Hospital Gynecologic Clinic, while clients were waiting for their medical records retrieved for periodic pelvic examination, we selected subjects who met admission criteria. Random sampling was applied to the selection. Ten clients a day were randomly selected with a separation of five numbers in between based on the list of wait numbers for examination. And then, we counseled selected subjects on study participation. Since waiting time was about 10-15 minutes, we realized that this short period was suitable for an interview. Subjects decided whether to join the study by themselves. Time for an interview was 15 minutes.

Step 3: Counseling on the study for client's agreement to sign consent form on study participation.

We invited the subject into the counseling room of the Gynecologic Clinic for individual discussion. There, we explained the study objectives as well as rights and benefits once she joined the study. The examination, treatment and health care procedures for clients still followed $\mathrm{Tu} \mathrm{Du}$ Hospital's guidelines. Based on this, the subject decided whether to participate in the study or not.

Step 4: Conducting interview and data collection

When agreed, clients signed a consent form to participate in the study. We conducted direct interview with them following the questionnaire to examine related factors. And then, we continued the discussion on the criteria table of insomnia diagnosis by DSM-5. If insomnia was detected, after interview, we counseled them on visiting a mental health hospital or Nguyen Tri Phuong Hospital Geriatrics and Mental Health Clinic for examination and treatment.

\subsection{Measurements / Instrument}

We defined an insomnia case per guidelines from DSM-5 (2015) [7-8]. A subject was considered an insomnia if all eight questions from "Insomnia-Disorder item" in "SleepWake disorder" chapter of DSM-5 were answered "Yes". Study subject's age was calculated as year of study minus year of birth. Economic status was a nominal variable with ordinal values of just enough for living or relatively wealthy that defined on subjective assessment.

\subsection{Statistical Analysis}

Statistical software (STATA 13.0) was used to analyze all the data. Data are presented as the mean \pm standard deviation (SD) and n (\%). For statistical analyses, a univariate analysis was done for independent variables to find out the association with the success of treatment. Then, we collected the variables which had $\mathrm{P}$ value $<0.2$ and completed a multivariate analysis. Confident interval of $95 \%$ was applied.

\subsection{Research Ethics}

Ethics approval for the study was obtained from the Institutional Review Board of TuDu Hospital on 16 Oct 2017 ( $\mathrm{N}_{0}$ : 2398/QD-BVTD). Informed consent was obtained from all patients.

\section{Results}

In our study, the average age was $48.1 \pm 2.27$. Regarding occupation, the group of freelance business took the highest part $(30.05 \%)$, the lowest was workers $(6.48 \%)$. The group of junior secondary education accounted for the largest proportion $(39.12 \%)$, the smallest was higher education $(13.73 \%)$. Most of the subjects had enough economic resources $(91.71 \%)$ (Table 1$)$. 
Table 1. Demographic and sociological characteristics of study subjects.

\begin{tabular}{lll}
\hline Characteristics & Total $(\mathbf{N}=\mathbf{3 8 6})$ & Proportion (\%) \\
\hline Age & & \\
\hline $45-49$ & 275 & 71.24 \\
$50-52$ & 111 & 28.76 \\
Occupation & & \\
Public employee & 75 & 19.43 \\
Farmer & 94 & 24.35 \\
Business & 116 & 30.05 \\
Worker & 25 & 6.48 \\
Unemployed, housework & 76 & 19.69 \\
Educational level & & \\
SPrimary & 80 & 20.73 \\
Junior secondary & 151 & 59.84 \\
Senior secondary & 102 & 86.27 \\
Higher & 53 & 13.73 \\
Economic status & & \\
Enough for living & 354 & 91.71 \\
Poor & 32 & 8.29 \\
\hline
\end{tabular}

The group with relatively regular menstruation had the highest part of $36.27 \%$. The proportion of hot flush was $39.64 \%$ and night sweat accounted for $24.09 \%$. The proportion of joint pain was $48.7 \%$. Vaginal dryness occurred in $49.78 \%$, and finally libido reduction was found in $58.55 \%$ (Table 2).

Table 2. Menstrual symptoms of study subjects.

\begin{tabular}{lll}
\hline Description & Total $(\mathbf{N}=\mathbf{3 8 6})$ & Proportion (\%) \\
\hline Menstrual status & & \\
\hline Regular & 140 & 36.27 \\
Long cycle $>7$ days & 38 & 9.84 \\
$\geq 2$ amenorrhea cycles & 137 & 35.49 \\
$\geq 12$ months' amenorrhea & 71 & 18.39 \\
Hot flush & & \\
No & 233 & 60.36 \\
Yes & 153 & 39.64 \\
Night sweat & & \\
No & 293 & 75.91 \\
Yes & 93 & 24.09 \\
Joint pain & & \\
No & 198 & 51.3 \\
Yes & 188 & 48.7 \\
Vaginal dryness & & \\
No & 217 & 56.22 \\
Yes & 169 & 49.78 \\
Libido reduction & & \\
No & 160 & 41.45 \\
Yes & 226 & 58.55 \\
\hline
\end{tabular}

As per DSM-5 criteria, there were 107 cases with insomnia accounting for $27.72 \%$ [ $95 \% \mathrm{CI}=23.31-32.47$ ], equivalent to $107 / 386$ cases (Table 3 ). Most of women with insomnia in line with DSM-5 insomnia criteria had complaints on onset insomnia (57.94\%). The majority who met diagnostic criteria had experienced insomnia for a period of 3-6 months (Table 4).

Table 3. Insomnia percentage by DSM-5 insomnia diagnosis criteria.

\begin{tabular}{llll}
\hline Insomnia & Total $(\mathbf{N})$ & Proportion (\%) & 95\%CI \\
\hline No & 279 & 72.28 & $67.53-43.69$ \\
Yes & 107 & 27.72 & $23.31-32.47$ \\
Total & 386 & 100 & \\
\hline
\end{tabular}

Table 4. Distribution of insomnia types.

\begin{tabular}{lll}
\hline Assessment items & \multicolumn{2}{c}{ Dissatisfaction of sleep time and quality } \\
\hline Insomnia types & \multicolumn{2}{l}{$57.94 \%$} \\
\hline Onset insomnia & 62 & $27.1 \%$ \\
Middle insomnia & 29 & $14.96 \%$ \\
Late insomnia & 16 & $100 \%$ \\
Total & 107 & \\
Insomnia period & & $69.16 \%$ \\
$3-6$ months & 74 & $26.17 \%$ \\
$6-12$ months & 28 & $4.67 \%$ \\
$12+$ months & 5 & $100 \%$ \\
Total & 107 & \\
\hline
\end{tabular}

Three factors were statistically significantly associated with insomnia; these are age, economic status and hot flush. Age group of 50-52 years increasing insomnia risk up to 1.92 times compared to age group of 45-49 years; group of poor economic status having insomnia risk of 2.99 times higher than that with sufficient economic status; group of hot flush raising insomnia risk up to 3.65 times higher than group without hot flush (Table 5).

Table 5. Multivariate analysis of subject's characteristics and insomnia.

\begin{tabular}{|c|c|c|c|c|c|}
\hline Characteristics & $\begin{array}{l}\text { Good sleep } \\
(N=279)\end{array}$ & $\begin{array}{l}\text { Insomnia } \\
(\mathrm{N}=107) \\
\end{array}$ & PR & $95 \%$ CI & $\mathbf{P} *$ \\
\hline \multicolumn{6}{|l|}{ Age } \\
\hline $45-49$ & $212(75.99)$ & $63(58.88)$ & 1 & & \\
\hline $50-52$ & $67(24.01)$ & $44(41.12)$ & 1.92 & $1.05-3.5$ & 0.034 \\
\hline \multicolumn{6}{|l|}{ Occupation } \\
\hline Public employee & $59(21.15)$ & $16(14.95)$ & 1 & & \\
\hline Farmer & $64(22.94)$ & $30(28.04)$ & 1.18 & $0.31-4.5$ & 0.804 \\
\hline Business & $90(32.26)$ & $26(24.30)$ & 1.06 & $0.3-3.76$ & 0,925 \\
\hline Worker & $14(5.02)$ & $11(10.28)$ & 3.67 & $0.8-16.58$ & 0.091 \\
\hline $\begin{array}{l}\text { Unemployed, } \\
\text { housework }\end{array}$ & $52(18.63)$ & $24(22.43)$ & 1.12 & $0.29-4.22$ & 0.868 \\
\hline \multicolumn{6}{|l|}{ Educational level } \\
\hline$\leq$ Primary & $55(19.71)$ & $25(23.36)$ & 1 & & \\
\hline Junior secondary & $99(35.48)$ & $52(48.6)$ & 1.72 & $0.79-3.73$ & 0.168 \\
\hline Senior secondary & $82(29.39)$ & $20(18.69)$ & 1.01 & $0.38-2.65$ & 0.988 \\
\hline Higher & $43(15.41)$ & $10(9.35)$ & 1.38 & $0.27-7.09$ & 0.7 \\
\hline \multicolumn{6}{|l|}{ Economic status } \\
\hline Enough for living & $263(94.27)$ & $91(85.05)$ & 1 & & \\
\hline Poor & $16(5.73)$ & $16(14.95)$ & 2.99 & $1.17-7.64$ & 0.023 \\
\hline \multicolumn{6}{|l|}{ Menstrual cycles } \\
\hline Regular & $115(41.22)$ & $25(23.36)$ & 1 & & \\
\hline$\geq 7$ days & $27(9.68)$ & $11(10.28)$ & 1.47 & $0.56-3.85$ & 0.436 \\
\hline $\begin{array}{l}\geq 2 \text { months of } \\
\text { amenorrhea }\end{array}$ & $90(32.26)$ & $47(16.85)$ & 1.65 & $0.84-3.22$ & 0.144 \\
\hline $\begin{array}{l}\geq 12 \text { months of } \\
\text { amenorrhea }\end{array}$ & $47(16.85)$ & $24(22.43)$ & 1.99 & $0.87-4.59$ & 0.103 \\
\hline \multicolumn{6}{|l|}{ History } \\
\hline No surgery & $276(98.92)$ & $100(93.46)$ & 1 & & \\
\hline $\begin{array}{l}\text { Unilateral } \\
\text { oophorectomy }\end{array}$ & $3(1.08)$ & $7(6.54)$ & 2.96 & $0.61-14.5$ & 0.179 \\
\hline \multicolumn{6}{|l|}{ Hot flush } \\
\hline No & $199(71.33)$ & $34(31.78)$ & 1 & & \\
\hline Yes & $80(28.67)$ & $73(68.22)$ & 3.65 & $2.01-6.6$ & 0.01 \\
\hline \multicolumn{6}{|l|}{ Night sweat } \\
\hline No & $230(82.44)$ & $63(58.88)$ & 1 & & \\
\hline Yes & $49(17.56)$ & $44(41.12)$ & 1.56 & $0.83-2.9$ & 0.166 \\
\hline \multicolumn{6}{|l|}{ Joint pain } \\
\hline No & $151(54.12)$ & $47(43.93)$ & 1 & & \\
\hline Yes & $128(45.88)$ & $60(56.07)$ & 1.16 & $0.6-2.02$ & 0.603 \\
\hline \multicolumn{6}{|l|}{ Vaginal dryness } \\
\hline No & $173(62.01)$ & $44(41.12)$ & 1 & & \\
\hline
\end{tabular}




\begin{tabular}{llllll}
\hline Characteristics & $\begin{array}{l}\text { Good sleep } \\
(\mathbf{N = 2 7 9 )}\end{array}$ & $\begin{array}{l}\text { Insomnia } \\
(\mathbf{N = 1 0 7 )}\end{array}$ & PR & 95\%CI & P* \\
\hline Age & & & & & \\
\hline Yes & $106(37.99)$ & $63(58.88)$ & 1.49 & $0.68-3.3$ & 0.316 \\
Libido reduction & & & & & \\
No & $128(45.88)$ & $32(29.91)$ & 1 & & \\
Yes & $151(54.12)$ & $75(70.09)$ & 1.03 & $0.45-2.4$ & 0.939 \\
\hline
\end{tabular}

(*) Multivariate regression

\section{Discussion}

Insomnia diagnosis by DSM-5 is currently applied around the world. Moreover, this tool has been demonstrated high sensitivity and specificity in insomnia diagnosis with values of $87.4 \%$ and $95.6 \%$ respectively $[12,14]$. In Viet Nam, especially in clinical practice at medical facilities without experienced mental health physicians, the application of DSM5 into insomnia diagnosis will help to standardize insomnia assessment and reduce errors in insomnia examination.

The study was conducted to analyze insomnia prevalence and related factors in perimenopausal women. Study data disclosed statistically significant relation between insomnia and the following factors: age group of 50-52 years increasing insomnia risk up to 1.92 times compared to age group of 45-49 years; group of poor economic status having insomnia risk of 2.99 times higher than that with sufficient economic status; group of hot flush raising insomnia risk up to 3.65 times higher than group without hot flush.

The risk of insomnia is lower than Ciano's 2017 in the United States [5]. Although both used DSM-5 criteria, the study in America was different since its variables contained information collected from the subject's medical history leading to difficult distinction between medical insomnia and stimulant insomnia.

Regarding age factor, some studies Shin et al, 2005 [15], Yazdi et al, 2013 [13] agreed with us when they concluded that insomnia rate increased with advanced age, but Simin Taavoni, 2015 [12] did not concur on this point. Considering economic status factor, we found correlation between insomnia and poor economic status. This result complied with Simin Taavoni et al 2015 [12] and Chol Sin, 2005 [13] but contradicted with Zohreh Yazdi, 2013 [13]. Our study concluded there was relationship between hot flush and insomnia, which went along with Zohreh Yazdi [13].

From 50 years of age on, most of women enter perimenopause and the decrease in level of reproductive hormones causes multiple symptoms resulting in insomnia risk. However, Simin Taavoni et al [12] believed there was no relationship between insomnia and age. The inconsistence may be due to difference in subject selection. Simin Taavoni (2015) [12] selected subjects aged 50-60 years whereas the variety of ages was seen in other studies. As per relationship between economic status and insomnia, this is a factor depending upon the study participant's subjective assessment. The variance among studies may be due to dissimilarities in poor or sufficient levels in various economies.

Results in previous studies often have some points for re- assessment. Firstly, the assessment tools of insomnia were not consistent across studies. In addition, the selection of related factors for analysis varied vastly. This does not allow a metaanalysis of factors and mechanism that led to insomnia. Ultimately, it is not able to fully rule out differences in research methodologies and data collection methods in various populations, which may lead to dissimilarities in results.

Our study still has some restrictions which did not survey representative population of Viet Nam. However, the study has achieved the initial objectives which defined insomnia rate and related factors in perimenopausal women who sought periodic pelvic examination at Tu Du Hospital.

The study disclosed that insomnia screening may be implemented in a simple way at medical facilities by medical interviewers who are trained in interview techniques. This is the principal purpose of comprehensive treatment strategy for patients. Given good screening and management, we can simply and radically treat illnesses related to premenopausal syndrome and menopause.

\section{Conclusion}

The prevalence of insomina in perimenopausal women at TuDu hospital was $27.72 \%$. The study results also found relationship between risk factors and insomnia in perimenopausal women. Therefore, perimenopausal women should be screened on insomnia when they contact medical staff for planning appropriate "all- sided" therapy to achieve the better quality of treatment. Moreover, the study also provides database to serve as a good source of reference for future studies.

\section{Acknowledgements}

We are indebted to the participants for making this research possible and to all physicians and staffs of Gynecology unit of TuDu hospital.

\section{Conflicts of Interest Declaration}

The author(s) have no conflicts of interest relevant to this article.

\section{References}

[1] Irwin M. R. (2015), "Why sleep is important for health: a psychoneuroimmunology perspective", Annu Rev Psychol, 66, pp. 143-72.

[2] Kelly J. M., Bianchi M. T. (2012), "Mammalian sleep genetics", Neurogenetics, 13 (4), pp. 287-326.

[3] Lampio L., Polo-Kantola P., Polo O., et al. (2014), "Sleep in midlife women: effects of menopause, vasomotor symptoms, and depressive symptoms", Menopause, 21 (11), pp. 1217-24.

[4] Abedi P., Nikkhah P., Najar S. (2015), "Effect of pedometerbased walking on depression, anxiety and insomnia among postmenopausal women", Climacteric, 18 (6), pp. 841-5. 
[5] Berek J. S. (2012), "Menopause", Berek and Novak's Gynecology 15th edition. Wolters Kluwer Health, pp. 21372153.

[6] Krystal A. D., Edinger J. D., Wohlgemuth W. K., et al. (2002), "NREM sleep EEG frequency spectral correlates of sleep complaints in primary insomnia subtypes", Sleep, 25 (6), pp. 630-40.

[7] Khurshid K. A. (2015), "A review of changes in DSM-5 sleep-wake disorders", Psychiatric Times, 32 (9), pp. 16-16.

[8] Sassoon S. A., de Zambotti M., Colrain I. M., et al. (2014), "Association between personality traits and DSM-IV diagnosis of insomnia in perimenopausal women: Insomnia and personality in perimenopause", Menopause, 21 (6), pp. 602-11.

[9] Ciano C., King T. S., Wright R. R., et al. (2017), "Longitudinal Study of Insomnia Symptoms Among Women During Perimenopause", Journal of Obstetric, Gynecologic \& Neonatal Nursing, 46 (6), pp. 804-813.
[10] Kravitz H. M., Joffe H. (2011), "Sleep during the perimenopause: a SWAN story", Obstet Gynecol Clin North Am, 38 (3), pp. 567-86.

[11] Kuh D., Hardy R., Rodgers B., et al. (2002), "Lifetime risk factors for women's psychological distress in midlife", Soc Sci Med, 55 (11), pp. 1957-73.

[12] Taavoni S., Ekbatani N. N., Haghani H. (2015), "Postmenopausal Women's Quality of Sleep and its Related Factors", J Midlife Health, 6 (1), pp. 21-5.

[13] Yazdi Z., Sadeghniiat-Haghighi K., Ziaee A., et al. (2013), "Influence of Sleep Disturbances on Quality of Life of Iranian Menopausal Women", Psychiatry Journal, 2013, pp. 5-10.

[14] Guidozzi F. (2013), "Sleep and sleep disorders in menopausal women", Climacteric, 16 (2), pp. 214-9.

[15] Shin C., Lee S., Lee T., et al. (2005), "Prevalence of insomnia and its relationship to menopausal status in middle-aged Korean women", Psychiatry Clin Neurosci, 59 (4), pp. $395-$ 402 . 